\title{
Delayed follow-up of medical retina patients due to COVID-19: impact on disease activity and visual acuity
}

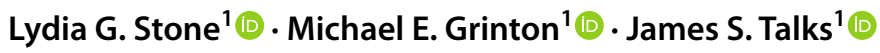

Received: 22 January 2021 / Revised: 12 March 2021 / Accepted: 23 March 2021 / Published online: 11 May 2021

(c) The Author(s), under exclusive licence to Springer-Verlag GmbH Germany, part of Springer Nature 2021

\begin{abstract}
Purpose The coronavirus pandemic has prompted unprecedented delays to treatment with anti-VEGF intravitreal injections due to the need to reduce hospital attendances and prioritise the patients at highest risk of vision loss. This study aims to quantify the effect of these delays on visual acuity (VA) outcomes and optical coherence tomography (OCT) features for patients receiving treatment for neovascular age-related macular degeneration (nAMD), retinal vein occlusions (RVO) and diabetic macular oedema (DMO) and correlate to the Royal College of Ophthalmologists guidelines (RCOphth).

Methods A retrospective data analysis of an electronic medical record was performed on a random sample of eyes receiving anti-VEGF injections for nAMD, RVO or DMO. Data collected included age, sex, reason for injection, number of weeks delay if $>8$ weeks from that planned, VA at baseline and follow-up and the OCT features, if delayed. For those eyes not delayed, a visual acuity at 20 weeks was recorded to provide a control group.

Results A sample of 981 eyes (858 patients) were analysed. There was a delay in review of 8 weeks or more in $39.6 \%$ of patients of which $30.4 \%$ had since returned for review (28.4\% nAMD, 37.6\% RVO and 30.0\% DMO). There was no demographic difference identified between the delayed and non-delayed patients; however, the delayed group was significantly more likely to have better vision in their non-treated eye $(p=0.0003)$. A statistically significant difference was found in the change in VA between the delayed and the not-delayed group for eyes with nAMD $(\mathrm{p}=0.001)$ but not for RVO or DMO. For the delayed group, mean CMT increased by 33 and $100 \mu \mathrm{m}$, respectively, for nAMD and RVO and decreased by $7.8 \mu \mathrm{m}$ for DMO. The VA of $89.7 \%$ of DMO eyes returned to baseline, compared to $74.6 \%$ and $76.9 \%$ of nAMD and RVO eyes.

Conclusion The RCOphth guidance to prioritise intravitreal injections for nAMD over DMO appears appropriate in this cohort but not for RVO. Eyes with nAMD experienced the greatest loss of vision with treatment delay, and nAMD and RVO eyes were less likely to return to baseline on restarting treatment.
\end{abstract}

\section{Key messages}

- The COVID-19 pandemic prompted unprecedented delays to treatment for patients receiving anti-VEGF intra-vitreal injections. This study has demonstrated that the Royal College of Ophthalmologists' guidance to prioritize treatment for those with neovascular age-related macular degeneration (nAMD) over diabetic macular oedema (DMO) was appropriate, however this was not true for retinal vein occlusion (RVO) patients. Patients with nAMD lost the most vision due to treatment delays, and it was less likely that their vision would return to baseline. The vision of RVO patients also did not recover to that measured prior to the delay. Central foveal thickness and vision did not change significantly in DMO patients with delay. Patients with better vision in the non-treated eye were more likely to be delayed.

Keywords Macular · Anti-VEGF · COVID-19 · Visual acuity

Extended author information available on the last page of the article 


\section{Introduction}

In December 2019, an outbreak of severe acute respiratory syndrome coronavirus 2 (SARS-CoV-2 or COVID-19), initiated in Wuhan China, spread worldwide [1]. The virus has severely disrupted health service provision resulting in a drastic reshape in healthcare infrastructure and provision.

In the height of the pandemic, ophthalmic healthcare services tried to minimise the number of patients attending hospital and only see those with a high risk of visual loss. Reducing hospital attendances was of particular concern in medical retina clinics where patients are often elderly and have comorbid conditions. In the United Kingdom (UK), the Royal College of Ophthalmologists COVID review team created medical retina management plans in response to this [2]. It advised considering maintaining neovascular age-related macular degeneration (nAMD) patients on 8-weekly anti-VEGF therapy and advised deferring anti-VEGF injections for most patients with diabetic macular oedema (DMO) and retinal vein occlusions (RVO). This has led some to predict that following these guidelines, approximately $25 \%$ of appointments would be delayed [3].

The pandemic has also caused high levels of psychological distress and anxiety, especially among patients with pre-existing health conditions [4]. Likely because of these high anxiety levels, despite many medical retina units prioritising seeing only those patients who were most at risk of irreversible visual loss, there were high levels of patient-initiated cancellations and 'did not attend' (DNA) rates [5]. There has not only been a reduction in outpatient clinic attendance, but one study also showed a more than 50\% reduction in emergency attendances [5].

Both randomised controlled trials and real-world data analyses have demonstrated the benefit of anti-VEGF injections in the treatment of medical retina conditions. Studies suggest that without intravitreal treatment, there will be a significant deterioration in patients' vision [6-9]. With this evidence, it can be predicted that the delays to anti-VEGF treatment attributed to COVID-19 will have a significant negative impact on visual outcomes.

With the onset of a 'second wave' of COVID-19 cases across Europe more recently, it is important to know the extent of the impact of delayed treatment and try to identify those patients at greatest risk of visual loss due to this delayed review. This study aims to provide real world data on the prevalence of delayed anti-VEGF treatment due to COVID 19 and the associated visual consequences.

\section{Methods}

All eyes who had at least one anti-VEGF injection at the Newcastle Eye Department from 1 January 2020 until 23 March 2020 (date of first UK national lockdown) for nAMD, RVO or DMO were identified. The following baseline data was recorded from a randomly selected sample of these patients: age and sex of patient, indication for anti-VEGF treatment, which eye(s) were receiving treatment, visual acuity (VA) of treated and untreated eyes and central macular thickness (CMT) of treated eye. Randomisation was carried out using the list randomisation function in Microsoft Excel (=RAND). Those patients in which the review appointment was delayed by 8 weeks or more (either due to postponement by the department or cancellation by patient) were then identified. In these delayed eyes, the following additional data were documented: VA at delayed review, CMT change since previous visit and OCT features (presence of intraretinal fluid and/or subretinal fluid). For those eyes where the review was not delayed, the VA at 20 weeks (or closest to this) after the baseline review was noted. This timeframe was chosen as this was the average time to review for the eyes which were delayed and was therefore seen as the most appropriate control measure. The majority of patients were on a treat and extend regime. The patients' electronic records were accessed again in November 2020, and it was noted whether the VA returned to within 5 letters of their baseline VA at any point since the delayed visit.

All data was recorded using an electronic medical record (EMR) system (Medisoft Ophthalmology; Medisoft Limited, Leeds, UK), which mandates collection of a standardised data set throughout a patient's care pathway. The lead clinician and Caldicott Guardian (nominee responsible for data protection) at the hospital gave written approval for anonymised data extraction. Anonymised database analyses of this type do not require ethical permission because they are viewed as audit or service evaluations (see http://www. hra.nhs.uk/research-community/beforeyou-apply/determinewhetheryourstudy-is-research/). This study was conducted in accordance with the Declaration of Helsinki and the UK's Data Protection Act.

Early Treatment Diabetic Retinopathy Study (ETDRS) VA letter scores at $2 \mathrm{~m}$ were recorded. Most VA values were recorded using habitual correction rather than with refraction as is usual in clinical practice. Values corresponding to counting fingers, hand movements, light perception and no light perception were substituted with values of 0 letters.

All participants had undergone imaging using spectral domain optical coherence tomography (SD-OCT) on the Heidelberg Spectralis (Heidelberg, Germany) in two centres under the care of Newcastle Eye Department.

Descriptive statistics were calculated using Excel 365 for Microsoft Office. The same software was used for production of graphics. Mean and median are reported to reduce the risk of a skewed mean result which might be seen if a few patients had a significant VA change. Statistical analysis was performed using Graphpad online. Unpaired $t$-tests were used to calculate statistical significance of the difference between the means for the delayed and non-delayed 
groups. Chi-squared statistic was used to compare the groups for categorical variables.

\section{Results}

Anti-VEGF injections had been given to 3255 eyes (2837 patients) between 1 January 2020 and 23 March 2020 for nAMD, RVO or DMO. Of these, 981 eyes (858 patients) were randomly selected, of which 681 (69.4\%) eyes (585 patients) had nAMD, 176 (17.9\%) eyes (173 patients) had RVO and 124 (12.6\%) eyes (100 patients) had DMO. This was comparable to the proportions in the original cohort for each disease indication (see Fig. 1).

\section{Delays}

Of the sample of 858 patients, $518(60.4 \%)$ were reviewed within 8 weeks of requested review, had died or had since been discharged. Seventy-nine patients $(9.21 \%)$ had yet to be seen, and 261 patients (30.4\%) had since been seen with a greater than 8-week delay (Fig. 1). This gives a total of 340 patients (39.6\%) delayed (36.6\% nAMD, $45.7 \%$ RVO and $47.0 \%$ $\mathrm{DMO})$. For the group who have been seen delayed, the mean delay was 13.1 weeks. In this group, 166 had nAMD (28.4\%), 65 had RVO (37.6\%) and 30 had DMO (30.0\%).

\section{Demographics}

For the whole cohort of patients, the mean age was 78.1 years (81.5 in nAMD, 75.0 in RVO and 63.9 in DMO), and $57.3 \%$ were female. For the randomised sample of 858 patients, the mean age was also 78.1 years (82.0 in nAMD, $76.1 \mathrm{RVO}$ and 61.3 in DMO), and $57.2 \%$ were female. For the patients who were delayed and subsequently seen, the mean age was 78.8 years compared to the non-delayed where the mean age was $78.4(p=0.650) ; 57.6 \%$ of eyes were female in the delayed group compared to the not delayed group where $56.9 \%$ of eyes were female $(p=0.840)$.

\section{Vision}

Table 1 shows the mean and median VA pre- and post-delay for the eyes of patients who were delayed by 8 weeks or more and in those whose review was not delayed.

Of the 298 eyes ( 261 patients) that were delayed and since seen, a delay of 8-16 weeks occurred in 234 eyes, and 64 eyes were delayed by over 16 weeks. For eyes delayed by $8-16$ weeks, VA changed from a mean of 61.5 letters (median 65) to 57.2 letters (median 62) compared to those eyes delayed by over 16 weeks who had a greater deterioration in VA from a mean of 56.3 letters (median 60) to 50.5 letters (median 55). Figure 2 shows the change in VA from

Fig. 1 Flow diagram demonstrating sample and the numbers of patients in delayed and not delayed groups

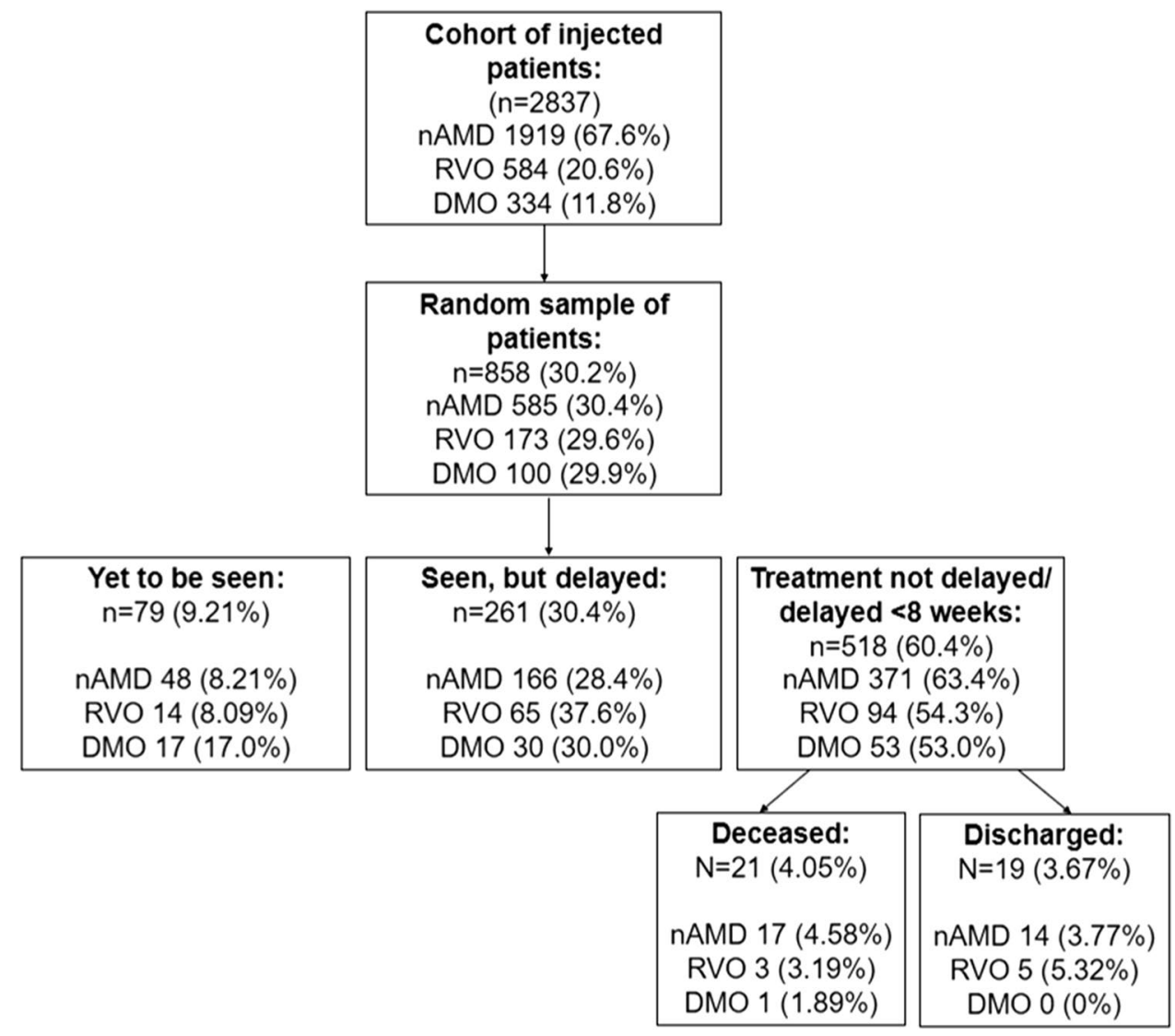



at baseline and at next review comparing those eyes delayed and not delayed and by diagnosis. (Review delayed $=$ next review was delayed by 8 weeks or more from what was requested by the clinician)
Table 1 VA (ETDRS letters)

\begin{tabular}{|c|c|c|c|c|c|c|c|c|}
\hline & \multirow[b]{2}{*}{ Number } & \multicolumn{2}{|c|}{ Baseline VA } & \multicolumn{2}{|c|}{ Review VA } & \multicolumn{2}{|c|}{ Change in VA } & \multirow{2}{*}{$\begin{array}{l}p \text { value } \\
\text { (change in } \\
\text { VA) }\end{array}$} \\
\hline & & Mean & Median & Mean & Median & Mean & Median & \\
\hline \multicolumn{9}{|l|}{ nAMD } \\
\hline Review delayed & 194 & 60.1 & 64 & 55.2 & 59 & -5.18 & -4 & 0.0010 \\
\hline Not delayed & 399 & 61.4 & 65 & 59.9 & 64 & -2.47 & -2 & \\
\hline \multicolumn{9}{|l|}{ RVO } \\
\hline Review delayed & 65 & 59.7 & 62 & 54.5 & 59 & -5.15 & -3 & 0.3627 \\
\hline Not delayed & 88 & 63.5 & 67 & 61.6 & 67 & -3.42 & -2 & \\
\hline \multicolumn{9}{|l|}{ DMO } \\
\hline Review delayed & 39 & 63.0 & 65 & 61.1 & 67 & -2.37 & +2 & 0.9780 \\
\hline Not delayed & 62 & 63.7 & 68 & 61.0 & 67 & -2.44 & -0.50 & \\
\hline \multicolumn{9}{|l|}{ All patients } \\
\hline Review delayed & $n=298$ & 60.4 & 64 & 55.7 & 60 & -4.82 & -4 & 0.0027 \\
\hline Not delayed & $n=549$ & 62.0 & 65 & 60.3 & 65 & -2.61 & -2 & \\
\hline
\end{tabular}

baseline to review split by diagnosis for both delayed and non-delayed groups.

For the 743 patients receiving unilateral anti-VEGF injections, the mean VA in the non-treated eye was 61.3 letters in the delayed group, compared to 53.3 letters in the nondelayed group $(p=0.0003)$.

\section{Disease activity on OCT}

Table 2 shows CMT data in those eyes which were delayed, split by diagnosis. Mean CMT for retinal vein occlusions increased by $+100 \mu \mathrm{m}$ in eyes with RVO compared to only +33 and $-7.8 \mu \mathrm{m}$ for $\mathrm{nAMD}$ and DMO, respectively. In order to reduce time in the department for patients due to coronavirus, some patients did not have vision and/or OCT at all visits, accounting for some missing data.

\section{Further analysis in nAMD eyes}

At the delayed review for eyes with nAMD, 74 eyes (38.1\%) had lost more than 5 letters compared to their baseline VA. The other 120 eyes (61.9\%) had maintained their VA (within 5 letters of their baseline). An OCT scan was available for 159 eyes at the delayed review, of which $43(27.0 \%)$ were dry, the rest having evidence of subretinal fluid, intraretinal fluid or both.
Fig. 2 Change in vision from baseline to review in those eyes delayed by 8 weeks or more (maintained vision is within 5 letters of baseline)

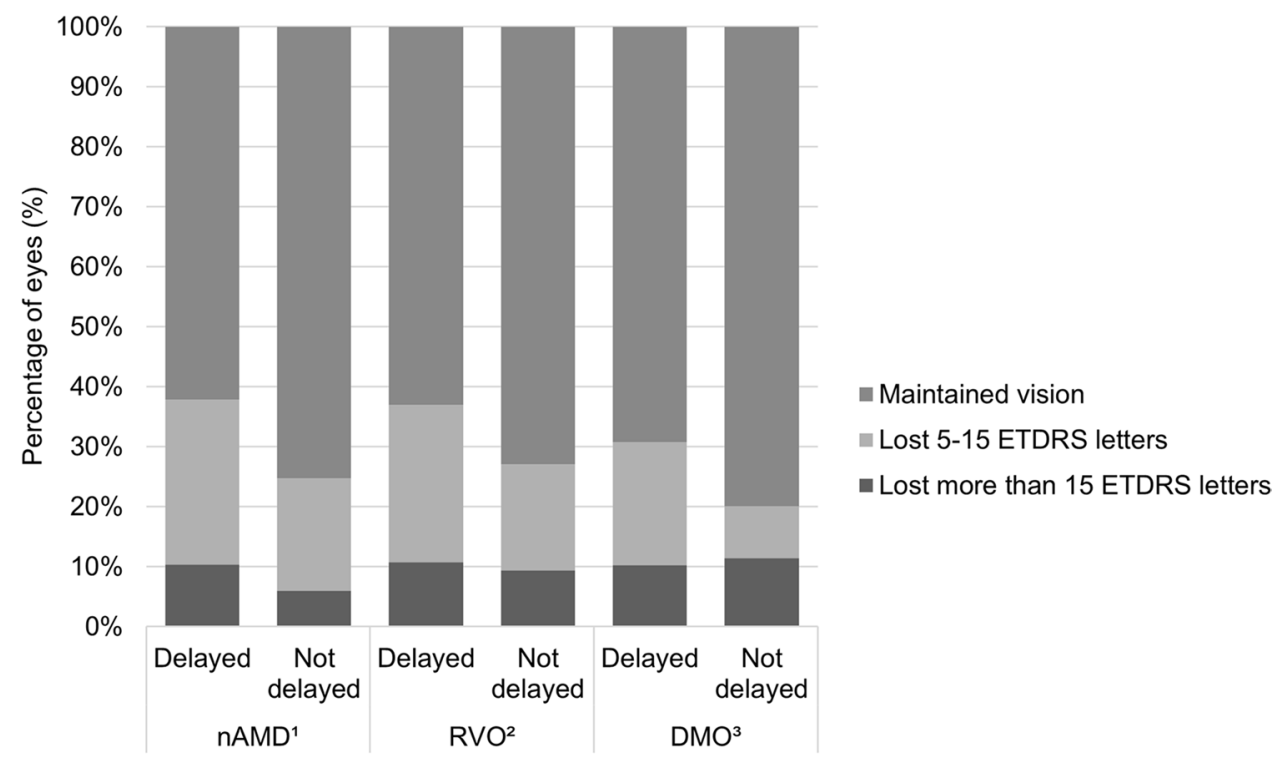

${ }^{1}$ nAMD Neovascular Age-Related Macular Degeneration

${ }^{2}$ RVO Retinal Vein Occlusion

${ }^{3}$ DMO Diabetic Macular Oedema 
Table 2 Central macular thickness (CMT) at baseline and at next review in those eyes whose review was delayed by 8 weeks or more

\begin{tabular}{|c|c|c|c|c|c|c|}
\hline & \multicolumn{2}{|c|}{$\begin{array}{l}\text { CMT at baseline } \\
(\mu \mathrm{m})\end{array}$} & \multicolumn{2}{|c|}{$\begin{array}{l}\text { CMT at delayed visit } \\
(\mu \mathrm{m})\end{array}$} & \multirow{2}{*}{$\begin{array}{l}\text { Mean change in CMT } \\
\text { for individual patient } \\
(\mu \mathrm{m}) \\
\text { Mean }\end{array}$} & \multirow{2}{*}{$\begin{array}{l}p \text { value } \\
\text { (change in } \\
\text { CMT) }\end{array}$} \\
\hline & Mean & Median & Mean & Median & & \\
\hline $\begin{array}{l}\text { nAMD } \\
(n=194)\end{array}$ & $\begin{array}{l}311 \\
(n=160)\end{array}$ & $\begin{array}{l}282 \\
(n=160)\end{array}$ & $\begin{array}{l}342 \\
(n=159)\end{array}$ & $\begin{array}{l}311 \\
(n=159)\end{array}$ & $\begin{array}{l}+33 \\
(n=151)\end{array}$ & 0.0074 \\
\hline $\begin{array}{l}\text { RVO } \\
(n=65)\end{array}$ & $\begin{array}{l}374 \\
(n=55)\end{array}$ & $\begin{array}{l}290 \\
(n=55)\end{array}$ & $\begin{array}{l}451 \\
(n=57)\end{array}$ & $\begin{array}{l}355 \\
(n=57)\end{array}$ & $\begin{array}{l}+100 \\
(n=52)\end{array}$ & 0.0375 \\
\hline $\begin{array}{l}\text { DMO } \\
(n=39)\end{array}$ & $\begin{array}{l}395 \\
(n=28)\end{array}$ & $\begin{array}{l}384 \\
(n=28)\end{array}$ & $\begin{array}{l}387 \\
(n=34)\end{array}$ & $\begin{array}{l}361 \\
(n=34)\end{array}$ & $\begin{array}{l}-7.8 \\
(n=28)\end{array}$ & 0.7878 \\
\hline $\begin{array}{l}\text { All delayed patients } \\
(n=298)\end{array}$ & $\begin{array}{l}335 \\
(n=238)\end{array}$ & $\begin{array}{l}292 \\
(n=238)\end{array}$ & $\begin{array}{l}373 \\
(n=254)\end{array}$ & $\begin{array}{l}327 \\
(n=254)\end{array}$ & $\begin{array}{l}+43 \\
(n=234)\end{array}$ & 0.0019 \\
\hline
\end{tabular}

Figure 3 shows OCT characteristics of eyes which maintained their VA at their delayed follow-up compared to those which lost more than 5 letters of VA. Eyes which have lost vision were more likely to have both intraretinal and subretinal fluid compared to those which maintained vision $\left(\chi^{2}[1\right.$, $n=159]=13.3, p=<0.05)$. Eyes which maintained vision were more likely to be dry compared to those eyes which lost vision $\left(\chi^{2}[1, n=159]=5.58, p=<0.05\right)$.

\section{Long-term VA}

On further notes review in November 2020, VA was within 5 letters of baseline in 229 of 297 eyes (77.1\%); one patient in this group was missing a follow-up VA due to expressive dysphasia. Figure 4 shows the proportion of eyes returning to baseline for each condition.

\section{Discussion}

In early 2020, during the height of the COVID-19 pandemic, healthcare services tried to minimise the number of patients attending hospital and only see those with a high risk of visual loss. For those clinicians treating patients in medical retina clinics in the UK, guidance was provided by the Royal College of Ophthalmologists COVID review team who created a medical retina management plan [2]. Some predicted that when following these guidelines approximately $25 \%$ of appointments would be delayed [3]. Despite limiting the
Fig. 3 Optical Coherence Tomography (OCT) characteristics of wet age-related macular degeneration eyes which maintained their visual acuity at their delayed follow-up compared to those which lost more than 5 letters of visual acuity

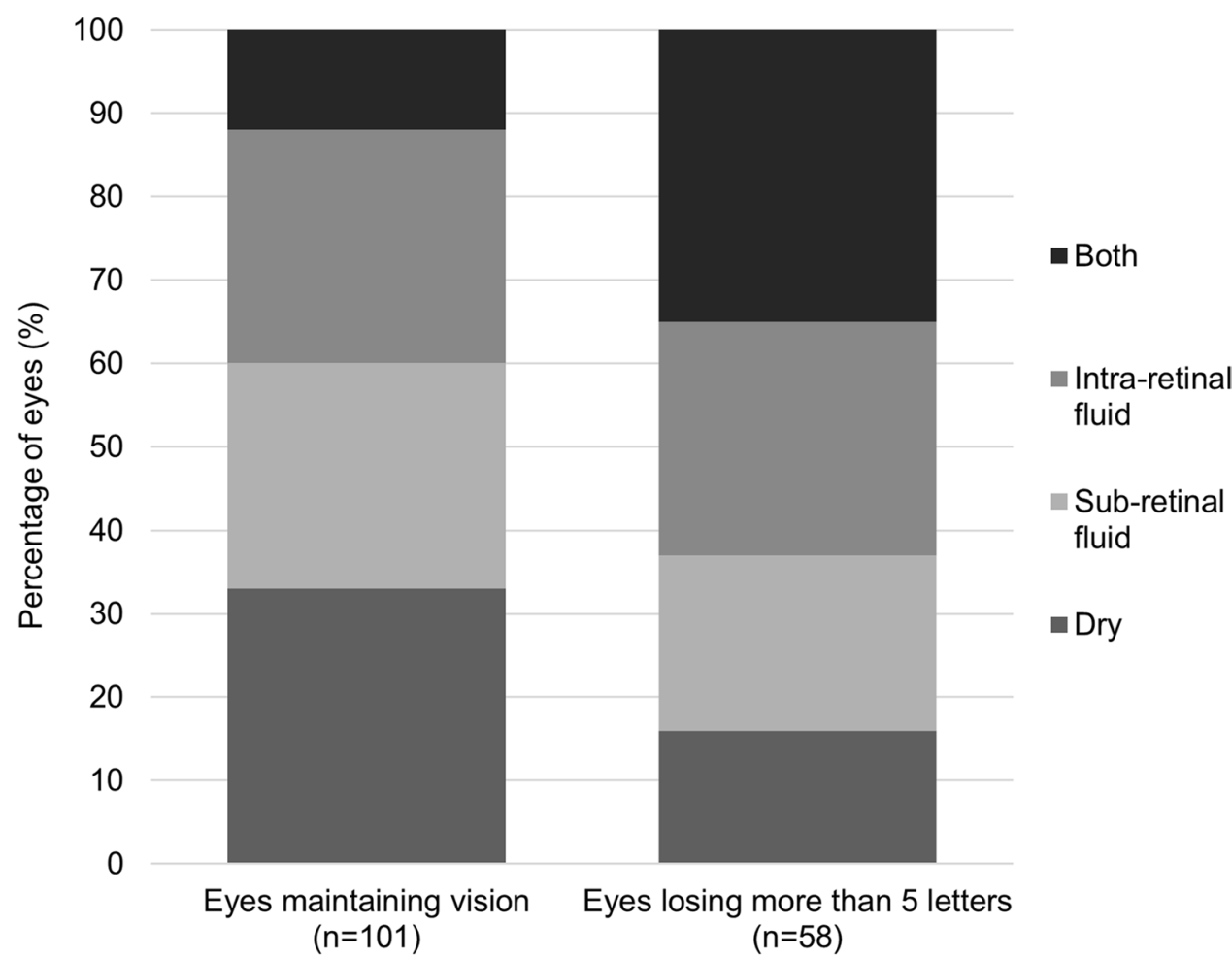


Fig. 4 Percentage of eyes returning to within 5 letters of their baseline visual acuity at any point since the delayed visit

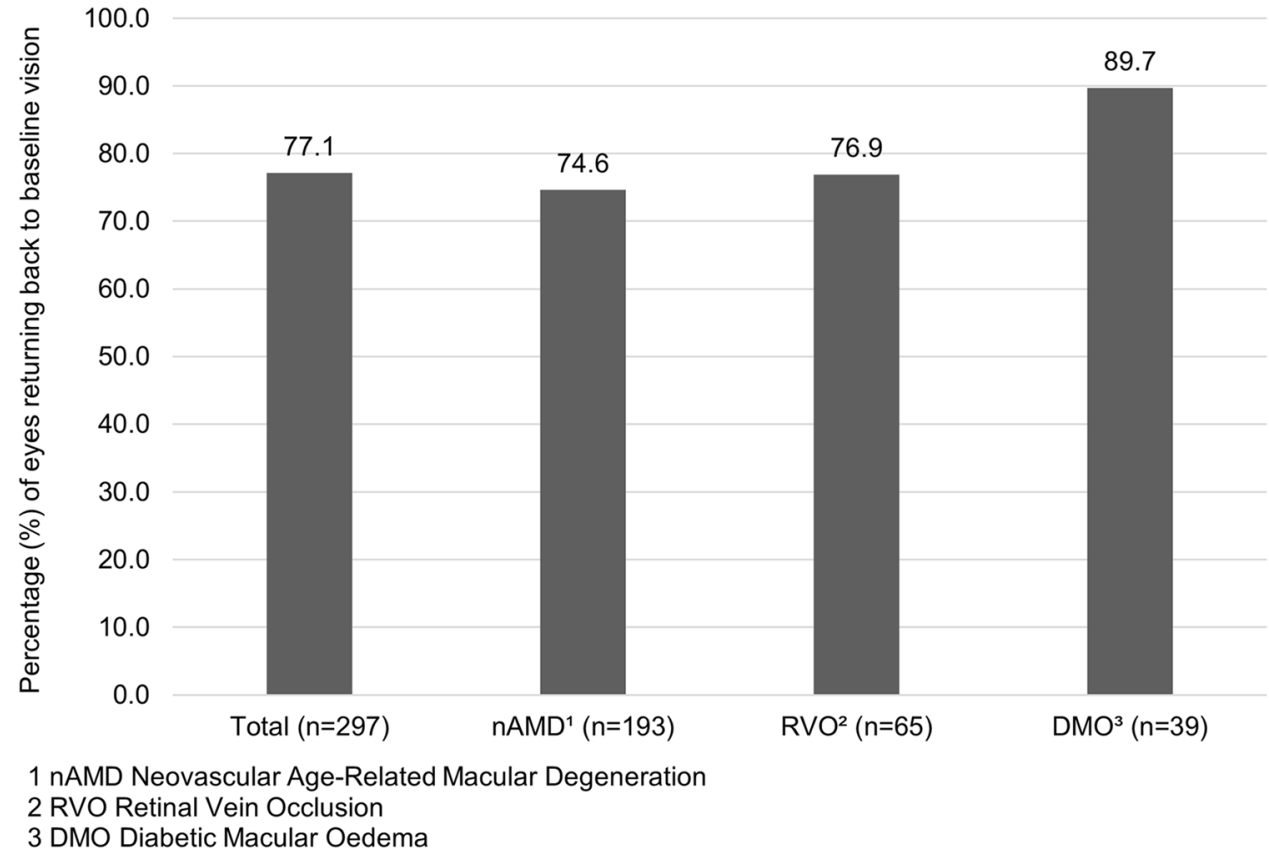

service to those patients most at risk of visual loss, most units have seen a larger reduction in activity than predicted, with multiple patient-initiated cancellations; one study showing the average DNA rate of $24.9 \%$ [5]. A study carried out in the USA reported a reduction in patient volume of $81 \%$ compared to 1 year ago, making ophthalmology the speciality with the greatest patient volume loss due to the COVID-19 pandemic [10].

Our study showed that of the 858 patients analysed, 340 (39.6\%) had review appointments delayed by 8 weeks or more. A higher proportion of eyes had a delay in the DMO (47.0\%) and RVO population (45.7\%) compared to nAMD (36.6\%). In the first 4 weeks of the UK lockdown, our unit only saw $33 \%$ of the patients booked for anti-VEGF review [3]. One study in Italy showed a $53.6 \%$ reduction in intravitreal injections, although this study showed a greater outpatient volume loss for AMD patients with a $79.9 \%$ reduction [11]. On analysis of which eyes were more likely to have their review delayed, there seemed to be no relationship with age and gender, but interestingly in those receiving unilateral anti-VEGF injections, better vision in the other eye resulted in higher chance of a delayed appointment. This may represent patients in whom the treated eye is the better eye or where the other eye also has pathology being more symptomatic or experiencing more anxiety. In our previous paper, we reported that of the patients receiving treatment to one eye, $57.6 \%$ were receiving treatment to their worse seeing eye [3]. Previous studies in patients receiving anti-VEGF injections have shown increased rates of non-attendance and being lost to follow-up in those with worse baseline vision and non-proliferative diabetic retinopathy (NPDR) stage in those with DMO [12]. For those with nAMD, older age, unilateral injections and living further away from the clinic were contributing factors [13, 14].

The Royal College of Ophthalmologists produced guidance as a pragmatic approach to maintain care for those patients who need it while deferring care for those patients who can wait. The guidelines prioritised certain conditions over others: advising 8-weekly injections for those with nAMD and advised considering deferring review for RVO and DMO. Guidance to prioritise injections in those with nAMD is based on the known poor outcomes of these patients prior to the advent of anti-VEGF. Multiple studies have shown worsening VA and OCT features in patients who have anti-VEGF injections delayed [15], with some studies suggesting poor VA persisting on final follow-up despite normalisation of central foveal thickness after a period of delayed treatment [16]. Our study does support the belief that nAMD eyes do indeed seem to be most at risk of visual loss with delays in anti-VEGF treatment but also shows that RVO eyes have an increased risk from treatment delay. Analysis of VA at the delayed appointment showed that nAMD and RVO eyes lost the most VA (a mean loss of 5.18 and 5.15 letters, respectively) compared to eyes with DMO ( 2.37 letters). Although the change in visual acuity for vein occlusions in the delayed compared to non-delayed group was not significant, the proportion of eyes who lost vision was similar to nAMD (36.9\% compared to $37.8 \%$ ), and we predict that with a bigger sample size this may have become significant. We also note that vein occlusion eyes had the greatest change in CMT. This visual loss continued more long term, with nAMD and RVO eyes being the least likely 
to return to their baseline VA once treatment had recommenced (74.6\% and $76.9 \%$ returning to baseline respectively, compared to $89.7 \%$ in DMO). Our study perhaps highlights that as well as maintaining injections for those with nAMD, more emphasis should be placed on maintaining RVO treatment as they lose comparable VA with delayed treatment and have similar longer-term visual outcomes. There is relatively little previous data on the effect of a lapse in treatment in those with RVO; however, some studies have shown worse outcomes in eyes where there has been a delay in the initial treatment with the anti-VEGF $[17,18]$. As well as having a drop in VA similar to nAMD eyes, eyes with RVO also had the largest increase in their CMT (mean change of +100 compared to +33 and -8 in nAMD and DMO, respectively). Visual loss was least in the DMO eyes which may have been predicted as evidence for lapses in anti-VEGF treatment is mixed; some showing a significant drop in VA [19] and others showing little effect on VA in the short term [20]. Our results show minimal drop in VA associated with delay in those with DMO and in fact a slight improvement in their CMT. The small sample size in eyes with DMO compared to nAMD and RVO should be noted, however.

Our study also highlights that in nAMD patients, a higherthan-expected number had dry OCTs despite having significant delays in their treatment. Despite the mean number of weeks delay being 13.1 weeks over the requested review, $26.7 \%$ of eyes remained dry. As expected, eyes with a dry OCT were more likely to maintain vision, compared to those losing more than 5 letters of vision which were associated with higher rates of intra- and sub-retinal fluid (Fig. 3). Although nAMD was, along with RVO, the condition most affected by delayed review, there is a subgroup within the nAMD population where substantial delays did not result in a drop in VA. The higherthan-expected proportion remaining inactive despite long delays suggests that these patients could perhaps have had more aggressive extension of their intravitreal treatment. The difficulty is with predicting which patients are most likely to remain dry and maintain vision, and we were unable to identify any OCT features prior to delay which helped predict these patients. As expected, eyes which maintained vision were more likely to have dry OCTs, but we also noted that those eyes losing vision were more likely to have both intraretinal and subretinal fluid. A previous study has provided evidence that patients treated with anti-VEGF who tolerated some SRF achieved VA that is comparable, with fewer injections, with that achieved when treatment aimed to resolve all SRF completely [21]. In our study, the proportion of eyes with only subretinal or intraretinal fluid were similar in those that maintained vision compared to those that lost vision.

A limitation of the study includes the relatively smaller number of DMO patients included in the analysis. Although this is a result of the random sample that was taken and represents the lower number of DMO patients seen in clinic compared to nAMD and RVO, this can potentially affect the accuracy of our analysis. Another limitation of the study is that when analysing the patients whose review was delayed, the reason for delay is not known. Although a significant proportion of delays are likely to have been patient initiated, there will have been a large number of patients who will have been delayed after notes had been reviewed by the clinician referencing the national guidelines. A small number of patients on subsequent review in clinic did not have repeat VA or OCT scans due to change in practice due to COVID-19 which again may have had a small impact on the accuracy of results. Lastly, although our study did involve review of patients EMR up to 8 months after the initiation of lockdown, we cannot make conclusions on the more long-term impact of delayed treatment on disease activity and visual outcomes.

In conclusion, we provide real-world data on how delayed follow-up of medical retina patients due to COVID-19 impacts on their disease activity and VA. Almost $40 \%$ of patients had their review delayed by 8 weeks or more, and we see that nAMD and RVO eyes were most affected by delayed review, with associated visual loss and worsening of activity on OCT scans. Despite some patients with nAMD having long delays there were a significant proportion which remained inactive, potentially highlighting that more aggressive extension of their treatment could have been attempted. The results of this study will help guide future decisions and guidance on antiVEGF treatment if disruption to treatment regimens occurs, and prioritisation of therapy is necessary in future.

Author contribution All authors contributed to the study conception and design. All authors contributed to data collection and analysis. The first draft of the manuscript was written by M E Grinton and all authors commented on previous versions of the manuscript. All authors read and approved the final manuscript.

Data availability Not available.

Code availability Not applicable.

\section{Declarations}

Ethics approval All data was recorded using an electronic medical record (EMR) system (Medisoft Ophthalmology; Medisoft Limited, Leeds, UK), which mandates collection of a standardised data set throughout a patient's care pathway. The lead clinician and Caldicott Guardian (nominee responsible for data protection) at the hospital gave written approval for anonymised data extraction. Anonymised database analyses of this type do not require ethical permission because they are viewed as audit or service evaluations (see http://www.hra.nhs.uk/ research-community/beforeyou-apply/determine-whether-your-studyis-research/). This study was conducted in accordance with the Declaration of Helsinki and the United Kingdom's Data Protection Act.

Consent to participate Not applicable.

Consent for publication Not applicable. 
Competing interests James S. Talks: Advisory board; Bayer; Novartis; Allergan; research; Bayer; Novartis; Allergan; Roche; Boehringer Ingelheim.

\section{References}

1. Cevik M, Bamford CGG, Ho A (2020) COVID-19 pandemic-a focused review for clinicians. Clin Microbiol Infect 26:842-847. https://doi.org/10.1016/j.cmi.2020.04.023

2. The Royal College of Ophthalmologists (2020) Medical retinal management plans during COVID-19. https://www.rcophth.ac.uk/ wp-content/uploads/2020/03/Medical-Retinal-Management-Planduring-COVID-19-UPDATED-300320-1-2.pdf. Accessed 29 Apr 2020

3. Stone LG, Devenport A, Stratton IM, Talks JS (2020) Macula service evaluation and assessing priorities for anti-VEGF treatment in the light of COVID-19. Graefe's Arch Clin Exp Ophthalmol 258:2639-2645. https://doi.org/10.1007/s00417-020-04849-8

4. Luo M, Guo L, Yu M, Wang H (2020) The psychological and mental impact of coronavirus disease 2019 (COVID-19) on medical staff and general public - a systematic review and meta-analysis. Psychiatry Res 291:113190. https://doi.org/10.1016/j.psych res. 2020.113190

5. Wickham L, Hay G, Hamilton R et al (2020) The impact of COVID policies on acute ophthalmology services-experiences from Moorfields Eye Hospital NHS Foundation Trust. Eye 34:1189-1192. https://doi.org/10.1038/s41433-020-0957-2

6. Kim JH, Chang YS, Kim JW (2017) Natural course of patients discontinuing treatment for age-related macular degeneration and factors associated with visual prognosis. Retina 37:2254-2261. https://doi.org/10.1097/IAE.0000000000001494

7. Browning DJ, Stewart MW, Lee C (2018) Diabetic macular edema: evidence-based management. Indian J Ophthalmol 66:1736-1750. https://doi.org/10.4103/ijo.IJO_1240_18

8. McIntosh RL, Rogers SL, Lim L et al (2010) Natural history of central retinal vein occlusion: an evidence-based systematic review. Ophthalmology 117:1113-1123. https://doi.org/10.1016/j. ophtha.2010.01.060

9. Saleh OA, Jammal H, Alqudah $\mathrm{N}$ et al (2020) Clinical experience in the administration of intravitreal injection therapy at a tertiary university hospital in jordan during the covid-19 lockdown. Clin Ophthalmol 24:2473-2480. https://doi.org/10.2147/OPTH.S269179

10. Strata Decision Technology (2020) Analysis: ophthalmology lost more patient volume due to COVID-19 than any other specialty. In: Eyewire. https://eyewire.news/articles/analysis-55-percentfewer-americans-sought-hospital-care-in-march-april-due-tocovid-19/. Accessed 21 Dec 2020

11. Borrelli E, Grosso D, Vella G et al (2020) Impact of COVID-19 on outpatient visits and intravitreal treatments in a referral retina unit: let's be ready for a plausible "rebound effect." Graefe's Arch Clin Exp Ophthalmol 258:2655-2660. https://doi.org/10.1007/ s00417-020-04858-7

12. Gao X, Obeid A, Aderman CM et al (2019) Loss to follow-up after intravitreal anti-vascular endothelial growth factor injections in patients with diabetic macular edema. Ophthalmol Retin 3:230-236. https://doi.org/10.1016/j.oret.2018.11.002

13. Obeid A, Gao X, Ali FS et al (2018) Loss to follow-up among patients with neovascular age-related macular degeneration who received intravitreal anti-vascular endothelial growth factor injections. JAMA Ophthalmol 136:1251-1259. https://doi.org/10. 1001/jamaophthalmol.2018.3578

14. Boulanger-Scemama E, Querques G, About F et al (2015) Ranibizumab for exudative age-related macular degeneration: a five year study of adherence to follow-up in a real-life setting. J Fr Ophtalmol 38:620-627. https://doi.org/10.1016/j.jfo.2014.11.015

15. Greenlee TE, Wang VY, Kang H et al (2020) Consequences of lapses in treatment with vascular endothelial growth factor inhibitors in neovascular age-related macular degeneration. Investig Ophthalmol Vis Sci 61:4202-4202. https://doi.org/10.1097/iae. 0000000000002888

16. Soares RR, Mellen P, Garrigan H et al (2020) Outcomes of eyes lost to follow-up with neovascular age-related macular degeneration receiving intravitreal anti-vascular endothelial growth factor. Ophthalmol Retin 4:134-140. https://doi.org/10.1016/j.oret.2019. 07.010

17. Lazić R, Boras I, Vlašić M et al (2010) Anti-VEGF in treatment of central retinal vein occlusion. Coll Antropol 34:69-72

18. Rezar S, Eibenberger K, Bühl W et al (2015) Anti-VEGF treatment in branch retinal vein occlusion: A real-world experience over 4 years. Acta Ophthalmol 93:719-725. https://doi.org/10. $1111 /$ aos. 12772

19. Weiss M, Sim DA, Herold T et al (2018) Compliance and adherence of patients with diabetic macular edema to intravitreal antivascular endothelial growth factor therapy in daily practice. Retina 38:2293-2300. https://doi.org/10.1097/IAE.0000000000001892

20. Yalamanchili SP, Maatouk CM, Enwere DU et al (2020) The short-term effect of a single lapse in anti-vascular endothelial growth factor treatment for diabetic macular edema within routine clinical practice. Am J Ophthalmol 219:215-221. https://doi.org/ 10.1016/j.ajo.2020.06.040

21. Guymer RH, Markey CM, McAllister IL et al (2019) Tolerating subretinal fluid in neovascular age-related macular degeneration treated with ranibizumab using a treat-and-extend regimen: FLUID Study 24-Month Results. Ophthalmology 126:723-734. https://doi.org/10.1016/j.ophtha.2018.11.025

Publisher's note Springer Nature remains neutral with regard to jurisdictional claims in published maps and institutional affiliations.

\section{Authors and Affiliations}

\section{Lydia G. Stone ${ }^{1}$ (] Michael E. Grinton ${ }^{1}$ (1) . James S. Talks ${ }^{1}$}

Lydia G. Stone

Lydia.stone1@nhs.net

$\triangle$ James S. Talks

James.talks@nhs.net
1 Newcastle Eye Centre, Royal Victoria Infirmary, Newcastle Upon Tyne Hospitals NHS Foundation Trust, Newcastle upon Tyne NE1 4LP, UK 